\title{
La formación de conceptos y símbolos en niños pequeños *
}

\section{Jonas Langer **}

Universitad de California, Berkeley'

Traduccion de Ileana Enesco

\section{Presentacion}

\section{Ileana Enesco $(*)$}

Universidad Complutense de Madrid

Jonas Langer es profesor del Departamento de Psicologia de la Universidad californiana de Berkeley. Discipulo de Werner y estudioso de la psicologia y epistemologia piagetianas, ba dedicado los últimos doce años de labor investigadora a estudiar los origenes de la lógica en la acción.

Langer es, sin duda, uno de los psicólogos americanos que mejor conoce a Piaget. Sus investigaciones sobre la protológica, como él la denomina - pues no se trata aun de la logica conceptual, sino de sus precursores en la acción-constituyen en alguna medida una prolongación de las investigaciones piagetianas sobre el periodo sensoriomotor y la inteligencia preoperatoria.

Su sólida formación teórica iniciada de la mano de Werner, junto con su rigor metodológico bacen de Langer una figura relativamente excepcional en el panorama de la psicologia americana. Desde su inspiración piagetiana no se ba limitado a una critica de la teoria de Piaget nacida de una mera contrastación de datos, como ba sido práctica común entre diversos psicólogos americanos. Por el contrario, Langer incorpora al estudio del desarrollo lógico temprano una poderosa metodologia que, a la vez que conserva los aspectos más importantes del método piagetiano en la fase de recogida de datos, enriguece el paradigma con nuevas formas de análisis de la acción. De su metodologia cabe resaltar dos aspectos fundamentales: la importancia que concede a las conductas espontáneas $y$, por otra parte, el tipo de análisis estructural que aplica a sus datos.

En este articulo Langer esboza parte de su teoria sobre el desarrollo lógico y simbólico a los dieciocbo meses. Aun cuando no ofrece, por prematura, una explicación definitiva de las relaciones entre ambos desarrollos, el articulo aventura algunas bipótesis sobre estas posibles relaciones. La posición implicita de Langer, apoyada por algunos de sus datos es que, con anterioridad a toda actividad representativa, existe una protológica con sus propias reglas estructurales más o menos sofisticadas. En torno a los dieciocho meses la actividad representativa dista aún mucho de poseer reglas tan complejas como las de la lögica de la acción. Es probable, incluso, que el desarrollo de la actividad representativa se apoye $y$ utilice en sus primeros estadios algunas de las reglas de

* "Concept and symbol formation by infants", 1983.

** Este articulo ha sido traducido y adaprado para su publicación en castellano por Ileana Enesco, en colaboración con el autor.

(") lleana Fnesco ha sido investigador ascociado al departamento de Psicología de la Universidad de Berkeley, California. Actualmente colabora con f. Langer en un proyecto de investigación sobre el desarrollo del espacio en bebés. 
la lógica de la accion, lo cual no significa que la relación entre el desarrollo representativa y el lógico sea de dependencia. Por el contrario, Langer sostient la bipotesis de que se trata de desarrollos independientes, con raices diferentes, reglas internas propias, pero con implicaciones mutuas.

Aparte de una extensa bibliografia en diversas revistas americanas y britanicas $y$ 'de su libro "Teorias sabre el Desarrollo", Langer tiene publicado ya un rolumen de sus investigaciones sobre desarrollo logico temprano. (From six to twelve). Este volumen, el primero de una serie de tres, trata el desarrollo de la protológica de los seis a los doce meses de edad. Su segundo volumen, que aparecerá el proximo año, continua con el desarrollo lógico durante el segundo año de vida. Por último, el tercer volumen iñcluirá el desarrollo lógico de los dos a los cinco años de edad, completando con el su ambicioso proyecto de investigación iniciado a principios de los setenta.

Actualmente, Jonas Langer sigue trabajando en el análisis de sus datos sobre la protológica y dirige diversas investigaciones nacidas de la aplicacion de su sistema a distintas áreas del desarrollo (espacial, numérico, físico, etc.). Además, su metodologia esta dando lugar a aplicaciones a desarrollos especiales como el de los. nivios autistas.

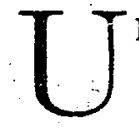

$\mathrm{N}$ problema para todas las grandes teorías sobre desarrollo cognitivo es determinar cuáles son las primeras relaciones evolutivas entre lenguaje y pensamiento (Piaget, 1923, 1945; Vygotsky, 1934; Werner, I948; Werner y Kaplan, 1963). Merece la pena considerar una vez más este problema por dos razones ligadas entre si. En primer lugar, análisis teóricos previos se han basado en datos en los que el desarrollo conceptual se confunde con el desarrollo simbólico. La segunda razón es que los datos microanaliticos derivados de nuestros estudios sobre desarrollo cognitivo constituyen nuevos descubrimientos sobre las construcciones conceptuales iniciales del niño. Además, estos datos son independientes de los obtenidos sobre la actividad simbólica infantil.

Aunque nuestros hallazgos se basan en datos obtenidos con sujetos entre seis y sesenta meses, el desarrollo a los dieciocho meses es crucial para el tema que nos ocupa, puesto que, como señalan investigadores de distintas orientaciones, es en este periodo cuando la actividad cognitiva infantil se convierte en representativa. Sin embargo, queda aún por determinar cómo se desarrolla la actividad cognitiva representativa durante este periodo y cual es su organizador estructural.

El análisis que presentamos a continuación se basa en nuestros datos sobre desarrollo conceptual, desarrollo simbólico y sus relaciones estructurales evolutivas a la edad de dieciocho meses. El lector puede encontrar nuestros resultados sobre desarrollo conceptual entre los seis y los dieciocho meses en otras publicaciones (Langer, 1980, 1981,1982 , en preparación). Por tanto, aqui me limitaré a analizar los hallazgos sobre el desarrollo conceptual a los dieciocho meses como paso previo al análisis de la formación del símbolo en esta edad.

El desarrollo conceptual adopta dos formas básicas: las operaciones parte-todo, que caracterizan el conocimiento lógico-matemático, y las funciones medios-fines, que caracterizan el conocimiento fisico. Revisaremos estos desarrollos conceptuales en las dos secciones siguientes.

El método utilizado para estudiar estas dos formas de desarrollo 
conceptual en bebés y niños pequeños es simple en la forma, pero complejo y elaborado en su ejecución. Se presenta a los sujetos pequeñas colecciones de diferentes objetos discretos y cuasi-continuos ' Los objetos están hechos de diferentes materiales: rígido, como la madera; dúctil, como la plastilina, y de combinaciones de ambos (por ejemplo, un palillo de manera con una bola de plastilina pegada en uno de sus extremos). El procedimiento comprende tres fases consecutivas de pruebas no verbales para elicitar una interacción manipulatoria con los objetos que se le presentan a los niños. La primera es una fase de actividad espontánea en la que los sujetos juegan libremente con los objetos. La segunda fase está diseñada para que los sujetos estructuren de alguna forma el material presentado. Por ejemplo, el experimentador puede empezar agrupando los objetos en dos clasés ante la vista del niño, incitándole a continuar la agrupación. La tercera fase incluye ciertas condiciones suplementarias; por ejemplo, contradicciones que consisten en presentar los objetos distribuidos en dos grupos numéricamente desiguales.

Los datos incluyen medidas cuantitativas de las manipulaciones espontáneas de los sujetos y de sus-construcciones con estos objetos, asi como observaciones cualitativas exhaustivas. Los resultados de las manipulaciones provocadas por el experimentador y de las contracondiciones complementan estas medidas.

Esta estrategia de investigación provoca transformaciones tanto en las relaciones parte-todo como en las relaciones medios-fines, aunque en el diseño experimental se ha intentado que predominen las primeras, puesto que estamos estudiando los origenes de la lógica. Pongamos un ejemplo de cada tipo de transformación: las relaciones parte-todo de un objeto dúctil de plastilina pueden transformarse modificando la forma del objeto, por ejemplo, a partir de un anillo hacer un objeto compacto, o a partir de un objeto compacto hacer un anillo, o ambas transformaciones. Un ejemplo del segundo tipo de transformación es cuando el bebé intenta colocar dos objetos adyacentes, uno encima de otro, transformando asi sus relaciones medios-fines desde la independencia hasta la dependencia espacial.

\section{LAS OPERACIONES}

He propuesto en otro lugar (Langer, i 980) que la organización del conocimiento lógico-matemático comprende tres grupos de estructuras de base. Estas son estructuras de transformaciones parte-todo que los sujetos producen al interactuar con su' entorno: (1) operaciones de combinatoria que incluyen las acciones de componer, descomponer y recomponer ${ }^{2}$; (2) operaciones relacionales de adición, sustracción, multiplicación y división, y (3) operaciones condicionales de intercambio (por ejemplo, la conmutatividad), de correlación (la reciprocidad) y de negación (por ejemplo, la correspondencia uno-a-uno). Los productos constructivos de estas operaciones son la equivalencia, la no-equivalencia ordenada y las relaciones reversibles entre elementos, conjuntos y series.

El desarrollo durante los primeros años de vida comprende dos niveles progresivos en la organización del conocimiento lógico-mate- 
mático. El primer año de vida está dominado por la formación de las operaciones combinatorias, relacionales y condicionales de primer orden.

Por ejemplo, a los seis meses de edad las interacciones del bebé consisten fundamentalmente en conductas de manipulación aplicadas a un solo objeto antes de pasar a otro. Estas conductas generan los rudimentos de: (a) las relaciones de emparejamiento que cumplen las mínimas exigencias de una equivalencia, es decir, correspondencias uno-a-uno y, menos frecuentemente, dos-a-dos (por ejemplo, golpear un objeto sobre la mesa dos veces seguidas, pausa, luego volver a golpearlo otras dos veces), y (b) la adición y sustracción pragmática, suficiente para producir series de orden iterativo que no excedan los dos puntos de magnitud (por ejemplo, golpear un objeto contra la mesa una vez, pausa, luego golpearlo dos veces seguidas).

-A los seis meses la mayöria de lós bebés limitan sus construcciones con objetos a composiciones muy lábiles y momentáneas, producidas por el bebé al manipular directamente sólo uno de los objetos (por ejemplo, cuando usa un objeto para empujar a otro). Algunas composiciones son un poco más avanzadas, como, por ejemplo, cuando el bebé manipula directamente los dos objetos estableciendo entre ellos una relación estable y no móvil.

Estas operaciones de primer orden siguen progresando durante el segundo año de vida, aunque empiezan a perder su primacia en el desarrollo cognitivo, dando lugar a las operaciones de combinatoria, relacionales y condicionales de segundo orden.

A los dieciocho meses los niños son ya capaces de reunir coherentemente hasta cuatro objetos en composiciones. Pueden también reunir coherentemente estas composiciones en recomposiciones derivadas de la original. Algunos de estos niños transforman sus composiciones de cuatro objetos en recomposiciones derivadas del mismo número de objetos, mediante las operaciones de sustitución, reposición y conmutatividad 3. Todas estas operaciones de intercambio producen una equivalencia cuantitativa entre composiciones y recomposiciones derivadas. $Y$ muchas de ellas se caracterizan por la reversibilidad por inversión.

Entre los seis y los dieciocho meses las operaciones de primer orden se desarrollan en dos sentidos: por una parte, su aplicación se extiende a un número cada vez mayor de objetos dentro de conjuntos y series simples; $y$ por la otra, estas construcciones se van volviendo progresivamente reversibles por negación. Todas estas operaciones de primer orden se limitan aún a producir equivalencias, no-equivalencias ordenadas y reversibilidad entre elementos y dentro de conjuntos; y series simples que el niño pequeño no relaciona ni cọordina con ninguna de sus otras construcciones.

La coordinación progresiva va a hacer posible el cambio de las operaciones de primer orden en operaciones de segundo orden. Las operaciones de segundo orden consisten en una coordinación de las operaciones de primer orden que desemboca en construcciones nuevas y más, poderosas. En realidad, consisten en coordinaciones de coordinaciones elementales. Su manifestación conductal más aparente es la coordinación simultánea, o con un breve desfase temporal, de dos series o conjuntos. 
A los dieciocho meses los niños son capaces de unir coherentemente un pequeño número de objetos en dos composiciones separadas, pero relacionadas entre si. Todos los niños, salvo raras excepciones, construyen dos conjuntos de dos objetos cada uno, en correspondencia uno-a-uno; por ejemplo, dos torres paralelas formadas por dos bloques cada una (Sugarman, 1982). Los sujetos de esta edad pueden también reunir estas composiciones binarias en recomposiciones binarias derivadas. Por ejemplo, casi la mitad de nuestros sujetos de dieciocho meses puede reunir sus composiciones binarias mediante una sustitución pragmática. Muchas de estas derivaciones por intercambio se caracterizan por la reversibilidad por inversión. Los productos de estas operaciones de intercambio de segundo orden son equivalencias cuantitativas derivadas o equivalencias de equivalencias. Por el contrario; los productos de las operaciones de intercambio de primer orden son sólo equivalencias cuantitativas elementales.

Estos descubrimientos ilustran un aspecto fundamental de la organización y desarrollo de la cognición lógico-matemática durante los primeros años de vida. Tanto los elementos constitutivos (las coordinaciones) del desarrollo estructural de las operaciones de-segundo orden como sus productos, son a la vez continuos y discontinuos respecto a las operaciones de primer orden, a partir de las cuales se desarrollan y a las que, a su vez, integran y coordinan entre si.

\section{LAS FUNCIONES}

En la organización y el desarrollo de la cognición física se esperan desarrollos paralelos. En torno a los dieciocho meses (a), los niños desarrollan funciones avanzadas de primer orden, y (b) desde el punto de vista estructural, las funciones de segundo orden empiezan a dominar el desarrollo.

Ambas sugerencias han sido confirmadas por nuestros hallazgos sobre las construcciones infantiles de las transformaciones causales medios-fines. Las transformaciones causales tienen un papel central en la formación de las primeras concepciones infantiles sobre los fenómenos fisicos.

Desde los seis meses los bebés generan dos formas rudimentarias básicas de funciones causales de primer orden. Una de ellas consiste en construir, replicar y observar efectos que dependen directamente de causas. Por ejemplo, los bebés pueden usar un objeto como un medio para empujar a otro repetidas veces, mientras observan sus construcciones causales. La otra forma complementaria consiste en anticipar y observar efectos que dependen de causas. Por ejemplo, los bebés pueden utilizar un objeto como un medio para cerrar el paso y detener a otro objeto que está rodando, mientras observan los efectos de sus predicciones y construcciones causales.

Hacia los dieciocho meses, los niños varian de forma semisistemática sus acciones sobre los objetos al construir funciones causales de primer orden. Los niños producen series ordenadas de acciones causales con el fin de provocar resultados diferenciales. Por ejemplo, empujan objetos cada vez con más fuerza y controlan y repiten 
cuidadosamente los resultados diferenciales. A esta edad los niños también varían las relaciones causales entre los objetos con el fin de controlar los efectos. Por ejemplo, empujan sólo un tipo de objetos para determinar los resultados.

Las funciones causales de primer orden son relaciones elementales de dependencia entre medios y fines. Formalmente, consisten en relaciones proporcionales direçtas entre semivariables causales independientes y dependientes, tales como «Desplazar Más Lejos es una función de Empujar Más Fuerten. Estas funciones relacionan sólo semivariables, porque causas y. efectos son variados de una forma todavia parcial y semisistemática. Asi, por ejemplo, los niños generalmente empujan sólo uno de los dos tipos de objetos que se les presenta y no intentan determinar los efectos diferenciales de empujar otro tipo de objetos.

Las funciones causales de segundo orden consisten en una coordinación de las transformaciones elementales medios-fines de primer orden. Formalmente, constituyen relaciones proporcionales entre semivariables causales independientes y dependientes, tales como «Desplazar (mover) es una función de Empujar, de igual modo que Detener es una función de Cerrar el Paso». Por tanto, los niños de dieciocho meses empiezan a covariar las relaciones de dependencia en sus transformaciones medios-fines. Por ejemplo, usan un objeto como instrumento para empujar a otro objeto dependiente; cuando el efecto conseguido es que el objeto se desplace rodando, convierten el instrumento en un medio para cerrarle el paso y detenerlo, y asi sucesivamente.

Así empiezan los niños a covariar las relaciones causales medios-fines en las funciones de segundo orden. Mientras que en las funciones de primer orden los fines dependen de los medios y éstos de los fines, pero sin coordinación entre ambos, las funciones de segundo orden coordinan las relaciones medios-fines mediante la covariación proporcional.

Por consiguiente, las funciones de segundo orden, al igual que las operaciones de segundo orden, son construcciones coordinativas que desembocan en cogniciones nuevas y más poderosas. También aquí los elementos constitutivos del desarrollo estructural de las funciones de segundo orden (la coordinación por covariación proporcional) y sus resultados (semivariables de equivalencia) son a la vez continuos y discontinuos respecto a las funciones de primer orden: se desarrollan a parrir de ellas, y, a su vez, las integran y coordinan entre sí.

\section{LA REPRESENTACION}

La literatura sobre formación del símbolo, incluido el desarrollo lingüistico, of rece poca información sobre su organización semántica y sintáctica cuando la actividad cognitiva infantil empieza a ser representativa. La lista de preguntas fundamentales sin respuesta es larga. ¿Las primeras estructuras subyacentes de simbolización; incluido el lenguaje, son semánticas, sintácticas o las dos cosas? ¿La semántica y la sintaxis tienen diferentes organizaciones en los primeros estadios del desarrollo lingüistico y simbólico? ¿Existe alguna diferencia entre las estructuras 
profunda y superficial de las relaciones gramaticales -como la de «sujeto de»- durante los primeros estadios del desarrollo lingüistico? $¿$ Las relaciones gramaticales forman parte del lenguaje infantil temprano? ¿Cuáles son, en caso de existir, las primeras relaciones entre las estructuras, cognitivas y las de la semántica y la sintaxis de la simbolización -incluido el lenguaje-? Es a esta última pregunta a la que quiero referirme a lo largo de las páginas siguientes.

En la medida en que la formación de conceptos y simbolos interactúa para dar lugar a la cognición representativa, hacia los dieciocho meses de edad, sugiero que existe una influencia predominante de la conceptualización sobre la simbolización. Esta propuesta es extensiva tanto: a la semántica como a la sintaxis de la simbolización.

Nuestra hipótesis de trabajo es que las operaciones y funciones de primer y segundo orden suministran las bases de los primeros estadios de la simbolización, incluido el lenguaje temprano, proporcionando un rico fundamento conceptual a la simbolización. Sólo una parte de la organización conceptual del niño de dieciocho meses es utilizada como rasgos semánticos por la simbolización incipiente.

Es en este sentido en el que las estructuras conceptuales infaritiles tienen implicaciones en la organización semántica de sus estructuras simbólicas en diferentes medios (gestos, imágenes y habla). Estas supuestas interacciones no son causales, sino implicativas. Estructuras semánticas que evolucionan independientemente (a), seleccionan significantes construidos mediante operaciones y funciones, y (b), los convierten en simbolos expresivos en diferentes medios. Las reglas que rigen la selección y la expresión figurativa, son propiedades inherentes a los sistemas simbólicos que, como tales, son estructuras que evolucionan independientemente.

Los hallazgos revisados en las secciones anteriores apoyan la tesis de que las estructuras conceptuales que se han desartollado hacia los dieciocho meses están formadas por construcciones de segundo orden relativamente complejas, como las funciones causales proporcionales. En comparación con esto, cualquiera de las expresiones simbólicas que acompañan estas acciones son muy limitadas. Generalmente, los niños sólo simbolizan un rasgo del suceso casual ( $18 B B$ y $18 P C$ ), o el objeto usado en la construcción causal ( $I 8 K M)$, o a la acción implicada en la transformación causal (1850):

$(I 8 B B)^{4}$ 1. Con ambas manos hace caer el Cilindro Rojo I, el Cilindro Rojo 2, y la Columna Exagonal Azul 2, que se hallaban de pie: Sujeto: "Boing"

(!8PC) 1. Con la mano izquierda empuja el Coche.

Sujeto: "Vroummm..."

(18KM) 1. Con la mano derecha hace rodar el Coche 4 de un lado a otro, dos veces seguidas.

Sujeto: "Car" ("Coche")

2. Con la mano derecha toca el Coche 3 .

$x=2$

3. Con la mano derecha hace rodar el Coche 3 de un lado a otro de la mesa, dos veces seguidas.

Sujeto: "Can"

(18SO) 1. Coge el Coche con la mano derecha e intenta ponérselo en la izquierda. El Coche se le cae. 
2. Con la mano derecha coge el Coche e intenta de nuevo colocarlo en su mano izquierda. El Coche se le cae.

Sujeto: "Drop"

Estas producciones simbólicas son típicas. A esta edad las causativas se limitan generalmente a sonidos onomatopéyicos o emisiones de una palabra. Sólo uno de los 12 sujetos de dieciocho meses produjo emisiones lingüisticas causativas más avanzadas; todas ellas acompañaban sus acciones de descomponer objetos de plastilina:

(1850) r. Con ambas manos rompe en dos piezas un trozo de plastilina que se halla a su izquierda.

Sujeto: «Broke it» («Lo rompi»)

2. Con la mano izquierda quita un trocito de plastilina del Anillo Circular t.

:- Sujeto: "I bbroke it. I brokew ("Yo lo rompi. Yo rompi»)

3. Con la mano izquierda arranca un trocito de plastilina de la Pelota 3 .

Sujeto: "Peel" ("Pelar")

4. Con la mano izquierda le arranca otro trocito de plastilina a la misma Pelota; luego un tercer trozo.

Sujeto: "Peeling" ("Pelando»)

5. Con la mano izquierda arranca un trozo de plastilina de la Pelota I, y asi sucesivamente hasta cuatro trozos.

Sujeto: "Doo. Ball" ("Doo. Pelota»)

Esta es la conducta lingüística más sofisticada producida por cualquiera de los sujetos de esta edad. El sujeto I 850 describe verbalmente sus descomposiciones mediante tres fases causativas ( broke it», «Peeling» y «Doo. Ball»). Incluso en este caso, este grupo de expresiones causativas no se aproxima a la complejidad de los significantes causales del niño de esta edad que, como hemos visto, incluyen funciones rudimentarias de segundo orden. Los niños generan cogniciones causales mucho más complejas de lo que podria suponerse si examinamos sólo sus producciones simbólicas causativas. Probablemente, por esto la comprensión lingüistica infantil es superior a sus producciones lingüísticas.

Nuestra hipótesis de trabajo sobre el desarrollo sintáctico inicial es análoga a la del desarrollo semántico. Las operaciones y funciones de primer y segundo orden constituyen una gramática estructural en desarrollo de las cogniciones elementales. Esta gramática estructural de la cognición prepara al niño para producir y comprender reglas de comunicación, tanto simbólicas como arbitrarias. Como tales, proporcionan los fundamentos además de facilitar la adquisición infantil de la sintaxis simbólica. Sin embargo, sólo una parte de este conocimiento gramatical básico es utilizada por la sintaxis simbólica, cualquiera que sea su medio de expresión, incluido el lenguaje en sus comienzos.

A los dieciocho meses, la gramática de la actividad cognitiva elemental incluye construcciones planificadas con todos los objetos que se les presentan a los niños (siempre que no sean muchos), construcciones que se realizan en contextos espaciales pequeños y cortos lapsos de tiempo. Estas construcciones suelen implicar aplicaciones (mappings) 5 binarias y ternarias (relaciones acción-objeto), bien diferenciadas entre si e integradas secuencialmente. Los resultados son rutinas relativamente bien diseñadas: 
(18MK) 1. Con la mano derecha coge el Vaso Amarillo y lo coloca de pie sobre la mesa.

A la vez, con la izquierda coge las Cucharas Roja y Amarilla.

2. Con la mano izquierda mete la Cuchara Roja en el Vaso Amarillo y la remueve rápidamente varias veces dentro del Vaso, golpeándolo; mientras tanto, mantiene la Cuchara Amarilla fuera del Vaso con la misma mano.

El Vaso Amatillo se cae como consecuencia de sus acciones.

3. Con la mano izquierda mete la Cuchara Roja en el Vaso Amarillo mientras mantiene la Cuchara Amarilla fuera del Vaso.

El sujeto aprieta el Vaso Amarillo contra su cuerpo.

4. Levanta las dos cucharas con la mano izquierda: mete la Amarilla en el Vaso Rojo y la mueve de un lado a otro varias veces mientras, con la misma mano, sujeta lä Cuchara Roja fuera del Vaso.

3. Sigue haciendo los mismos movimientos que en (4) con ambas cucharas, pero ahora lo hace fuera del Vaso, sobre la mesa, aunque sin golpearla, es decir, con movimientos al aire.

6. Mete la Cuchara Roja en el Vaso Rojo con la mano izquierda, manteniendo la Cuchara Amarilla fuera del Vaso.

7. Saca la Cuchara Roja del Vaso Rojo mientras mantiene la Amarilla fuera del Vaso con la misma mano. A la vez, con la mano derecha, pone de pie el Vaso Amarillo.

8. Con la mano izquierda mete la Cuchara Roja en el Vaso Rojo manteniendo la Amarilla fuera del Vaso.

El Vaso Rojo se cae.

Con la mano derecha toca el Vaso Amarillo.

Estas rutinas bien organizadas representan sólo un pequeño fragmento de las transacciones sistemáticas de $18 \mathrm{KM}$. Están precedidas y seguidas por varias repeticiones y variaciones sobre un tema básico: mantener de pie los dos vasos con una mano, de forma que con la otra puede meter (colocar, mover o presionar) una cuchara en un vaso mientras mantiene la otra fuera del vaso.

Durante la construcción, la única relación aparente es que las transacciones ${ }^{6}$ se generan simultáneamente (lineas 7 y 8). Sin embargo, es evidente que el sujeto ha preparado las condiciones necesarias para una coordinación operatoria sucesiva:

(18MK) 9. Con la mano izquierda mete la Cuchara Roja dentro del Vaso Amarillo, sin mirar; a la vez sujeta la Cuchara Amarilla fuera del Vaso.

10. Con la misma mano mueve de un lado a otro la Cuchara Roja dentro del Vaso Amarillo, sin mirar, mientras sigue mantenien-
do la Cuchara Amarilla fuera del Vaso.

Los resultados son inserciones sucesivas y repetitivas de la misma cuchara en los dos vasos diferentes; de este modo se van formando correspondencias reversibles en coordinación. con sustituciones reversibles.

A los dieciocho meses, la sintaxis de la acción se caracteriza por dos tipos de desarrollo. El primero consiste en la elaboración de un pequeño conjunto de aplicaciones relativamente nuevas, como la acción de «insertar" (meter). Algunas de estas nuevas aplicaciones están 
marcadas por rasgos simbólicos convencionales o semiconvencionales (por ejemplo, $18 M K$ ).

El segundo desarrollo consiste en la combinación de aplicaciones elementales en rutinas de aplicación pequeñas, aunque coordinadas entre sí, como las acciones de "coger un objeto, cambiarlo de mano y coger otro", y las acciones de "meter un objeto en un vaso mientras sujeta otro $y$, con la otra mano, pone de pie otro objeton. La integración secuencial de estas acciones en formas regidas por reglas empieza con la coordinación de un número muy pequeño de aplicaciones elementales en combinaciones breves, pero bien diseñadas, es decir, en rutinas protosintácticas ${ }^{7}$ cortas: Estas se repiten varias veces convirtiéndose en rutinas bastante bien practicadas y regidas por reglas; y se aplican a todos los objetos siempre que su número sea pequeño.

Las rutinas de aplicación de los niños pequeños no presentan muchos rasgos que marquen completamente las construcciones sintácticas; por ejemplo, carecen de una organización jerárquica. Sin embargo, están ya marcadas por otros rasgos sintácticos, como es la interrupción que se manifiesta clara y repetidamente en las rutinas de aplicación de $18 M K$ (Goodson y Greenfield, 1975). A pesar de estar regidas por reglas, estas rutinas son aún protosintacticas, porque están construidas pór el individuo y pueden (por ejemplo, cuando se mete la cuchara en el vaso) o no (por ejemplo, cuando coge sucesivamente objetos con una mano y los va pasando a la otra mano) ajustarse a las reglas convencionales o semiconvencionales de la conducta simbólica.

Además, las reglas de las rutinas protosintácticas son menos permanentes, y probablemente están mucho menos constreñidas (o tienen más grados de libertad) que las producciones sintácticas completas. Como tales, son construcciones más idiosincráticas y menos eficientes, como sistemas potenciales de comunicación, que los sistemas simbólicos sintácticos completos. En consecuencia, son menos susceptibles a la retroalimentación social necesaria para que sean elaborados en sistemas simbólicos completos de comunicación.

Una consecuencia de estas transacciones protosintäcticas es que el dominio semántico construido por los niños pequeños empieza a ampliarse exponencialmente. Los niños pequeños ya no construyen el dominio semántico de transacciones como si representara simplemente formas elementales, como «objetos que pueden ser empujados» u «objetos que pueden ser sujetados». Los niños construyen el dominio semántico de manera que puedan ya incluir combinaciones protosintácticas de formas elementales, tomando generalmente dos formas a la vez $e$ incluso tres. Estas rutinas rudimentarias de aplicación suponen extensiones reconstructivas y anticipatorias de las representaciones infantiles inmediatas en representaciones mediatas. Asi pues, constituyer las condiciones evolutivas estructurales necesarias para que el aqui-y-ahora de las aplicaciones protosimbólicas se transforme en el no-aqui-y-no-ahora de los sistemas simbólicos o lenguajes.

El dominio semántico sigue siendo protosimbólico en la medida en que las representaciones construidas por los niños de este estadio no están todavia totalmente separadas de los objetos de sus transacciones, no son aún construcciones arbitrarias $y$ no son aún sistemas de comunicación totalmente convencionales. Así pues, el dominio semán- 
tico se convierte en simbólico como una función de la progresiva flexibilidad del niño en combinar aplicaciones en pequeñas rutinas marcadas por rasgos rudimentarios protosintácticos, reproductivos $y$ anticipatorios.

Los rasgos simbólicos convencionales y semiconvencionales marcan progresivamente (a) aplicaciones que ya se han generado en este estadio ( $18 B B$ ), incluso en estadios anteriores, y (b) nuevas aplicaciones que no estaban presentes en estadios previos ( $I 8 K M)$ :

( $\$ 8 B B$ ) I. Con la mano derecha se cepilla el pelo (de delante atrás) con la parte trasera del Cepillo, mientras sonrie (is segundos).

2. La misma operación, esta vez utilizando un Espejo.

3... Con su propia mano (derecha) se cepilla el pelo (de atrás adelante) durante ocho segundos aproximadamente.

4. Vuelve a cepillarse el pelo, pero esta vez utiliza realmente el Cepillo por el lado correcto (mano derecha).

3. Con la mano derecha coge la Cuchara y se cepilla el pelo con ella.

(18KM) x. Coge una Cuchara con la mano derecha y la utiliza como una pala, haciendo como si cavara en una Caja Grande que contiene varios objetos. Repite la acción dos veces.

2. Coge otra Cuchara con la mano izquierda y realiza la misma operación: hace como si cavara dentro de la misma Caja.

Como puede observarse, el sujeto $18 B B$ sustituye objetos convencionales (un cepillo) y semiconvencionales (un espejo, una cuchara; su propia mano) como medios funcionales equivalentes para cepillarse el pelo, de forma convencional (de delante atrás) o semiconvericional (de atrás hacia delante).

Las construcciones protosintácticas marcan también nuevas formas de aplicación:

(18BB) 1. Con la mano derecha saca la Muñeca Verde que estaba metida boca abajo en el Vaso Naranja 2. El Vaso Naranja 2 se encuentra en la parte superior de una torre, construida previamente por el sujeto, compuesta por cuatro vasos (Naranja I/Amarillo I/Amarillo 2/Naranja 2).

2. Con ambas manos coloca la Muñeca en posición correcta y la pone sobre la mesa, a la derecha de la torre de vasos.

3. Coge el Vaso Naranja 2 por el borde (con la mano derecha) y lo saca de la torre de vasos.

4. Con la misma mano acerca el Vaso hacia sí mismo y lo inclina en dirección a la boca. Mientras, mira dentro del vaso.

s. Acerca el.Vaso a sus labios y hace movimientos de labios como si bebiera.

6. Mantiene el Vaso con ambas manos a la altura de la boca, pero alejándolo de la cara.

7. Lo sujeta con ambas manos encima de la mesa, sin tocarla.

8. Con la mano derecha inclina el Vaso hacia la Muñeca como si le echara agua encima, mientras sonrie.

Con la otra mano sujeta la Muñeca acercándola al borde del vaso que mantiene inclinado con su mano derecha.

9. Coloca el Vaso de pie sobre la mesa, con la mano derecha.

10. Con la izquierda, mete la Muñeca dentro del Vaso.

i1. Levanta el Vaso con la Muñeca dentro y lo inclina hacia si mismo, mirando dentro del Vaso. 
estadio. Incluye ajustes preparatorios (líneas 1-4) y conductas simbólicas, como la de simular beber del vaso (linea s) y simular echar agua sobre la muñeca (línea 8). Así pues, estas rutinas protosintácticas incluyen muchos elementos de conductas de simulación flexibles. Por ejemplo, esta rutina supone transformat la conducta de simulación de beber liquido de un vaso vacio a la de echar líquido del mismo vaso vacio sobre una muñeca.

Estas construcciones se hallan a mitad de camino entre la protosimbolización y la simbolización verdadera. Como hemos visto, algunas implican una sustitución lúdica, que es una forma de construir aplicaciones de aplicaciones (Werner y Kaplan, 1963). Las rutinas de aplicación lúdicas (como simular echar líquido de un vaso vacio sobre una muñeca) empiezan repreșentando aplicaciones reales (como.echar realmente líquido de un recipiente sobre un objeto). Estas conductas están marcadas por sustituciones de primer orden (por ejemplo, un vaso vacio por uno lleno). Algunas de estas rutinas lúdicas protosintácticas empiezan ya a sustituir y emparejar (por correspondencia uno-a-uno) objetos arbitrarios y objetos prototipicos (Fein, 1975):

(18BB) I. Con la mano derecha se cepilla el pelo utilizando una Columna Triangular, durante diez segundos.

A la vez, con la otra mano, realiza la misma operación utilizando otra Columna Triangular, durante el mismo tiempo.

A veces estas rutinas lúdicas de aplicación empiezan incluso, aunque de una forma muy rudimentaria, sustituyendo órganos del cuerpo por instrumentos convencionales. (Kaplan, 1968; Overton y Jackson, 1973). Recordemos como ejemplo que $18 B B$ usa su mano y su brazo como sustitutos de un cepillo (linea 3 del primer protocolo de $18 B B$ preseñtadö en página 13 ).

Estas construcciones semisimbólicas, como hemos visto, están marcadas por dos rasgos importantes. En primer lugar; son protosintácticas. Comprenden rutinas de aplicación ordenadas secuencialmente y regidas por reglas. Por una parte, estas rutinas están marcadas por la regularidad de la repetición. Sus repeticiones se hallan constreñidas a copias bastante exactas. Por otra parte, estas rutinas de aplicación están marcadas por alguna flexibilidad. y pequeñas arbitrariedades. Por ejemplo, diferentés medios pueden ser sustituidos entre si (como espejos, cucharas y cilindros, por cepillos) dentro: de una rutina de aplicación dada. Estos objetos son transformados en una clase de equivalencia de "Cepillos» mediante funciones de primer orden. Dé este modo se van separando progresivamente como formas protosimbólicas.

El segundo rasgo importante que marca a la semisimbolización es la convencionalización de las aplicaciones. Por una parte, estas aplicaciones se generan en concordancia con el nivel cognitivo operatorio y funcional de los niños. Por otra parte, las aplicaciones se van adecuando progresivamente a las propiedades de uso convencional de los objetos, como las conductas de simular comer o mezclar con una cuchara. 


\section{LA.COGNICION REPRESENTATIVA}

Durante este estadio, la sintaxis de la simbolización en acción (por ejemplo, las rutinas de $I \$ B B$ de simular beber o verter líquido de un vaso vacio) es al menos tan compleja como la sintaxis potencial de la simbolización mediante palabras, aunque pueden existir algunas raras excepciones a esta asincronia evolutiva estructural, como señalábamos antes. A los dieciocho meses, la longitud media de las emisiones lingüisticas es de una palabra (Brown, 1973) y su significado es holofrásico (Werner y Kaplan, 1963). Agente, acción y objeto son diferenciados y coordinados (en series, no jerárquicamente) mediante símbolos en rutinas de acción, mientras que, desde el punto de vista

$\therefore$ semántico o sintáctico, esto no es posible mediante las emisiones dé una palabra.

Durante la segunda infancia y en los años posteriores, la sintaxis de la simbolización en palabras alcanza y supera con creces a la simbolización en la acción. Cómo y cuándo ocurre esta inversión evolutiva entre los simbolos en la acción y los símbolos en palabras, constituyen : problemas aún no resueltos y que requieren mucha investigación empírica y análisis teóricos. Una hipótesis sobre este proceso, es que la simbolización primitiva en palabras empieza utilizando y aprovechándose de la avanzada simbolización en acción. Esto podría resolver el. problema de cómo la sintaxis verbal se iguala a la sintaxis de la acción:pero no podría explicar cómo la sintaxis verbal supera a la sintaxis de la acción. Ello requeriria postular la emergencia de un mecanismo que está aún sin determinar. De acuerdo con esta hipótesis, deberían proponerse procesos totalmente diferentes para dar cuenta de las fases en las que el desarrollo lingüistico «alcanza» primero y luego «supera» a la sintaxis de la acción. Por el momento, esta solución teórica no es elegante ni económica.

La única producción verbal que hemos encontrado a los dieciocho meses comparable en "nivel de complejidad gramatical a los simbolos en acción, fue generada por un niño, 1850 (véase el fragmento del protocolo en la pág. 9). Frases como "Lo rompi. Yo lo rompi» ("Broke it. I broke it») y «Yo rompi» («I broke») son mucho más complejas de lo que usualmente se observa a esta edad (Braine, 1963; Brown, 1973). Además, los análisis lingüísticos hábituales no permiten determinar en qué medida las estructuras subyacentes a estas producciones verbales reflejan reglas semánticas sobre el agente, la acción y el paciente, o reglas sintácticas sobre el sujeto, el verbo y el objeto, o ninguna de estas reglas (Bowerman, i978). En cualquier caso, estas construcciones lingüisticas sofisticadas emitidas por un niño no exceden la complejidad m. gramatical de los símbolos en acción generados por la mayoria de los niños de dieciocho meses.

Tampoco debemos perder de vista el hecho de que la estructura de . las funciones de segundo orden incluye ya relaciones conceptuales relativamente complejas entre agentes, acciones y objetos. Como hemos visto, sus relaciones están ya regidas por esquemas proporcionales. $\mathrm{Ni}$ nuestros datos ni ninguno que yo conozca de la literatura sobre el tema, permiten insinuar la posibilidad de que, a los dieciocho meses, la simbolización esté regida por reglas sintácticas que se aproximen a la complejidad y semisistematicidad de la proporcionalidad rudimentaria. 
No hay ninguna prueba de que durante este estadio la simbolización en cualquier medio de expresión, incluido el lenguaje, pueda aprovechar los resultados de una conceptualización tan avanzada.

Obsérvese, además, que las estructuras de las cogniciones fisicas forman una sola pieza; constituyen una organización unificada de una forma fundamental: las funciones. Del mismo modo, las cogniciones lógico-matemáticas adoptan una forma fundamental: las operaciones. En oposición a la unidad de la formación de conceptos, la formación de símbolos varía, a menudo radicalmente; según el medio de expresión; desde los símbolos gráficos y motores (como en el dibujo y en los gestos) hasta los más arbitrarios y estipulados (como el habla y; especialmente, las matemáticas). Esta relación de uno-a-varios entre la conceptualización y la simbolización explica, al menos en parte, el desfase inicial entre la formación de simbolos y el desarrollo conceptual.

En este periodo de edad, cuando los niños empiezan a tener una actividad representativa, se pueden dar cuatro tipos de interacción entre la formación de conceptos y la de símbolos. Las dos primeras relaciones posibles son simétricas. La formación de conceptos y la de símbolos pueden ser sistemas de desarrollo estructural dependientes que se influyen mutua e igualmente. Alternativamente, pueden ser sistemas estructurales totalmente independientes que no tienen ninguna relación de implicación entre si. Entre estos dos extremos existen dos relaciones asimétricas posibles entre la formación de conceptos y la de símbolos. En ambos casos, los sistemas estructurales son independientes, pero la formación de conceptos puede tener consecuencias implicatorias para la formación de símbolos, y/o la formación de símbolos puede tener consecuencias implicatorias para la formación de conceptos.

Nuestros datos sólo son coherentes con la tercera y cuarta posibilidades. Como hemos visto, el desarrollo de las operaciones lógico-matemáticas elementales (de primer y segundo orden) y de las funciones físicas no depende del desarrollo de la actividad simbólica. Correlativamente, no hemos encontrado pruebas que indiquen que la formación de simbolos dependa de la formación de conceptos; aunque nuestros datos no permiten desechar de una forma directa esta posibilidad.

Aunque la formación de conceptos y la de simbolos constituyen sistemas estructurales independientes, el desarrollo de uno tiene implicaciones sobre el desarrollo de otro. Por ejemplo, hemos observado que la construcción de conceptos fisicos elementales evoluciona independientemente de la formación de simbolos. En efecto, la cognición física en desarrollo precede a la formación de simbolos sobre fenómenos físicos. En consecuencia, la cognición proporciona a la simbolización una creciente organización conceptual del significado de lost fenómenos físicos, como la covariación entre semivariables causales y dependientes. La organización semántica y sintáctica del lenguaje temprano, asi como otros medios simbólicos, utiliza sólo un subconjunto de la organización conceptual del niño pequeño. $Y$ : si, por hipóteșis, la concepción precede a la simbolización, entonces debería esperatse -y de hecho se ha encontrado- que los simbolos físicos (como las causativas) sean más primitivos que los conceptos físicos (como las funciones causales) durante esta etapa formativa de la 
El desarrollo de la simbolización elemental tiene también implicaciones en la formación de conceptos. La simbolización puede ser utilizada por los niños pequeños para facilitar y ampliar las operaciones $y$ funciones elementales. Por ejemplo, hemos observado que las conductas simbólicas de juego de simulación permiten la sustitución de un objeto presente y arbitrario (por ejemplo, una columna) por uno no presente y prototípico (por ejemplo, un cepillo). Por tanto, la simbolización amplia el rango computacional de las funciones y operaciones. $\mathrm{Y}$ lo hace al menos de dos formas básicas. En primer lugar, multiplica los elementos constantes de las operaciones y funciones. En segundo lugar, aumenta el espacio sobre el cual se aplican las operaciones y funciones.

En cónsecuencia, nuestros resultados apoyan la hipótesis de que la transformación de las operaciones y funciones de primer grado en operaciones y funciones de segundo grado inicia el desarrollo estructural de la representación elemental. La simbolización amplía la representación elemental extendiéndola hasta el no-aquí y no-ahora. De este modo estamos empezando a determinar cómo se desarrolla la cognición representativa y cuál es su organización estructural.

1 Se han utilizado tres tipos de objetos diseretos: (a) objetos geométricos compactos, como columnas triangulares, cilindros; (b) objetos geométricos anulares (ring objects), como anillos, triängulos, etc., y (c) objetos realistas, como eucharas, muñecas en miniatura, coches, etc., de distintos colores. Entre los objetos cuasj-continuos, hechos de un material muy flexible y fácilmente rompible, semejante a la plastilina, se han utilizado dos formas análogas a las de los objetos discretos: (a) formas compactas, como balones y cubos, y (b) formas agujereadas (anulares) como anillos y triángulos.

2 La composición se aplica tanto a objetos directos como a objetos cuasi-continuos, aunque sús resultados son radicalmente diferentes. La composición de objetos discretos consiste en la construcción de conjuntos o colecciones de elementos. La composición de elementos cuasi-continuos (como utrozos) de plastilina) resulta en la construcción de un único objero.

La acción inversa es la de descomponer, esto es, destruir un conjunto previamente construido (por ejemplo, mediante la sustracción de los elementos uno a uao) o descomponer un objeto cuasi-continuo en varias partes (por ejemplo, troceándolo). Recomponer consiste en trañsformar las relaciones parte-todo de una colección de objetos o de un único objeto cuasi-continuo (por ejemplo, mediante la sustitución de un elemento del conjunto por otro o de una parte del objeto por otra) formando una nueva composición derivada de la anterior.

3 Por sustitución se entiende la acción de quitar un objeto del conjunto y colocar otro en su lugar. Por reposición, la acción de quitar un objeto y volverlo a colocar en el mismo lugar que ocupaba. Conmutatividad es la acción de colocar un objeto en la posición de otro que, a su vez, es desplazado a la posición del primero:

- En los fragmentos de protocolos que se citan a lo largo del articulo los sujetos son identificados por su edad en meses y por sus iniciales. $18 B B$ es un sujeto de dieciocho meses, cuyas iniciales son BB. EI número que precede a cada linea indica cada conducta o transacción del sujeto.

5 Las aplicaciones (mappings) son funciones que expresan las consecuencias transformacionales de las acciones sobre los objetos; en particular, las aplicaciones son una forma de caracterizar cómo las àcciones transforman las relaciones parte-todo (a) dentro de un único objeto y (b) entre dos o más objetos. El concepto de aplicación es más amplio que el concepto de esquema. Este último se refiere sólo a los procesos y productos del sujeto, mientras que el concepto de aplicación ineluye además los procesos y productos de las selaciones entre cl sujeto y los objetos.

6 Existen tres formas de interacción transformacional: integrativa, coordinativa y transactiva. La transacción se refiere a las interacciones entre las estructuras funcionales del sujeto y el medio fisico y social, es decir, interacciones entre sujeto y objeto. La integración y la coordinación se refieren a las interacciones internas al propio sujeto, dentro de una misma estructura funcional o entre distintas estructuras (por ciemplo, estructuras fisicas y lógicas).

7 Hemos adoptado el prefijo "proton para dejar claro que la actividad lingüistica avanzada no es reducible a sus antecedentes, es decir, a la simbolización pragmática precursora del lenguaje. 


\section{Resumen}

En este articulo el autor analiza el desarrollo conceptual y simbólico y sus relaciones evolutivas a los dieciocho meses. De acwerdo con los datos que aporta el autor sobre el desarrollo conceptual temprano, existe un desfase entre el desarrollo simbolico y al conceprual siendo la formación del simbolo un fenomeno evolutivo de aparición posterior. Los datos apoyan la bipotesis de que la formacion de conceptos y de simbolos constitugen sistemas estructurales independientes aunque ambos desarrallos tienen implicaciones mulwas. Por una parte, la cognición proparciona una organización conceptual relativamente sofissicoda que sera utilizada, aunque sólo en parte, por la temprana simbolización. Por otro lado, la simbolización amplia el espacio sobre al que se aplica. la conceptualización extendiendolo basta el no-aqui y no-ahora.

\section{Summary}

In this paper the developmental. relations at the age of 18 monshs between conceptual and symbolic development are analysed. Based on bis data on early conceptial development the aufor maintains tbat there is an initial lag of symbol formation bebind conceptual development. The data support the bypothesis that conceptual and symbol formation are independent structural systems that bave implicatory muival consequences. On one band, cognition provides a relatively sophisticated conceptual organization wbicb is used, to a small degree, by carly symbolization. On the otber band, symbolization increases the space to wbicb conceptualization applies, by extending it to tbe not-herc and the not-now.

\section{Résumé}

Dans cet article lauteur analyse le developpement conceptuel et symbolique et leurs relations à lage de 18 mois. Les donnees aporties para rauteur sur le developpement conceptuel montrent un décalage entre La formation du symbole ef celle des concepts, etan! la symbolisation d"apparition plus tardive. Les donnees appuitent Ibspotbise suivante: la formation du symbole et le developpement conceptuel constituent des systemes structuraux independants ayant, cependant, d'implications mutuelles au cours ds developpement. D'une part, la cognition fournit une organisation conceptuelle relativantent sopbistiquee qui sera utilisée en partie para la symbolisation initialle. D'autre part, la symbolisation elargit respace sur lequel s'applique la concepfualisation, en Fitendant jusqu'ax non-ici et non-maintenant.

\section{Referencias}

BowERMAN, M.: "Structural relationships in children's utterances: Syntactic or semantic?". En L. BLOOM (Ed.): Readings in language development. New York: Wiley, 1978.

BRAINE, M. D. S.: "The ontogeny of English phrase structure: The first phrase." Language, 1963, 39, 1-13. BrowiN, R.: A first-language: The early stages. Cambridge: Harvard University Press, 1973.

FEIN, G. A.: “A transformational analysis of pretending". Developmental Psychology, $1979,11,291-296$ :

G(x)DSCN, B., y GREENFIEI.D, P.: «The search for structural principles in children's manipulative play: A parallel with linguistic developments Child Development, 1979, 46, 734-746.

Kaplixi, E: Gestural representation of implement usage: An organismic developmental study. Tesis doctoral incidita, Clark University, 1968.

LANGER, J.: The origins of logic: Six to twelue months. Nueva York: Academic Press, 1980.

LANGER, J.: Logir in infancy. Cognition, $198 \mathrm{t}, 10,18 \mathrm{1}-186$.

LANGER, J.: «From prerepresentational to representational cognition». En G. Forman (Ed.), Action and thought. Nueva York: Academic Press, 1982.

LANGER, J.: The origins of logit: One to two years. En preparacion.

OVERTON, W. F. \& JACKONS, J. P.: "The representation of imagined objects in action sequences: $A$ developmental study". Child Development, 1973, 44, 309-314.

PIAGET, J.: Play, dreams and imitation in childbood. Nueva York, Norton, 1945, 1951. Trad. cast. La formación del simbolo en el niño. México: F.C.E., $196 \mathrm{I}$.

PIAGET, J.: The language and thought of the child. Nueva York: Meridian, 1926, 195s. Trad. cast. El lenguaje $y$ el pensamiento en el niño. Buenos Aires. Guadalupe, 1972.

Stigarmai, S.: "Transitions in early representational intelligence". En G. FORMaN (Ed.), .1ction and thought. Nueva lork: Academic Press, 1982.

VycotsKY, L. S.: Thought and language. Cambridge: MIT Press, 1934/1962. Trad. cast. Pensamiento y lenguaje. Buenos Aires. I-a Plèyade, 1973.

WERner, H.: Comparative pjebology of mental development. Nueva York. International Universities Press, 1948. Trad. cast. Psicologia comparada del desarrollo mental.

Werver, H., y Kinı.AN, B.: Symbol Formation. Nueva Jork, Wiley, 1963. 